\title{
Dynamic and static deuterium inventory in ASDEX Upgrade with tungsten first wall
}

\author{
V. Rohde, M. Mayer, V. Mertens, R. Neu, K. Sugiyama \\ and the ASDEX Upgrade Team
}

May 29, 2009

e-mail contact of main author: Volker.Rohde@ipp.mpg . de

\begin{abstract}
Hydrogen retention in the divertor tokamak ASDEX Upgrade is studied by surface analysis and gas balances methods. Comparing carbon and tungsten plasma facing components (PFCs), the deuterium content of deposits at the divertor plates has dropped by a factor of 13 . With tungsten PFCs only $0.7 \%$ of the puffed hydrogen is retained, including a significant amount deep implanted in the tungsten coatings at the outer divertor. Gas balances for ITER relevant high density H-mode discharges leads to a hydrogen retention averaged over a discharge of $8.2 \pm 3.3 \%$ for tungsten PFCs and $23 \pm 7 \%$ for carbon PFCs. For tungsten PFCs wall saturation is observed, i.e. only $1.5 \pm 3.5 \%$ of the puffed gas is retained after reaching steady state conditions. Within the accuracy of the gas balance measurements all hydrogen outgasses within $15 \mathrm{~min}$.
\end{abstract}

PACS numbers: 52.25.Fi, 52.25.Ya, 52.55.Fa 


\section{Introduction}

First wall materials of future fusion reactors have to match different requirements. Plasma facing components (PFCs) have to withstand thermal loads, the erosion rate must be low to allow ample operation time, the dilution of and the radiation in the core plasma must be tolerable and the storage of hydrogen must be tolerable. Present ITER design tries to match the different requirements at different location using a mix of beryllium, tungsten and carbon fibre composite (CFC) surfaces. An alternative to this material mix is a complete high $\mathrm{Z}$ wall. This option is tested in ASDEX Upgrade (AUG) by using tungsten for PFCs. A stepwise transition from carbon to tungsten coated carbon tiles was selected to minimize the effort and to study material migration [1],[2],[3].

For ITER the total amount of tritium on the site will be restricted by the licensing [4], and a strong retention and accumulation of tritium will be not allowed due to safety reasons. Hydrogen in a fusion device is stored by different mechanisms: implantation in the bulk material, diffusion and trapping at natural or ion induced traps, co-deposits with eroded PFCs (such as carbon and beryllium) and adsorption or solution at the surface [5]. Cleaning of implanted hydrogen is difficult as it is bound inside the material, but for safety reasons a higher inventory is tolerable. In co-deposits, typical for carbon PFCs, hydrogen is chemical bound in layers on the surface of the tiles. Hydrogen can be released by heating up the surface or by removal of the hydrogen containing surface layer, as for example is observed when these layers are flaking off (producing dust). Co-deposits are the dominant safety risk, as in carbon devices they store most of the hydrogen. Absorption and solution are located on the surface, hydrogen is released within some hours in case of adsorption or even faster for solution [6]

In this paper we discuss two methods to measure the hydrogen retention in AUG. The first idea is to measure the hydrogen content of the PFCs. A set of new 
tiles and probes was build in, exposed and post mortem analyzed by ion beam techniques [7]. The advantage of this method is the high sensitivity of the analysis, but the problem is to cover all deposition areas. As the tiles are analyzed after the campaign, the result is a weighted average of all different kinds of discharges. The other possibility is the gas balance method, which determines for each discharge the gas input and gas removal. Unfortunately the retained gas is the difference of large quantities: an adequate accuracy has to be reached. No information on the mechanism and position of the retained gas is gathered. Both techniques are complementary and therefore needed to get a consisted picture of the hydrogen retention.

\section{Deposition on Plasma Facing Components}

In the divertor tokamak AUG the dominant plasma wall interaction is located at the lower divertor. Stripes of thin coatings from different materials are used on the divertor tiles as markers. Erosion and deposition was determined from the thicknesses of the markers before and after a campaign and the deposition on top of them [8],[9]. A set of tiles, covering the complete cross section of the divertor, was replaced after each campaign to study the stepwise transition to a full tungsten device [7]. The carbon source plays an important role for the hydrogen retention, as hydrogen is bound in a: $\mathrm{CH}$ layers formed during carbon re-deposition. The primary carbon sources are hard to identify by spectroscopy, as carbon recycles manifold at the main chamber [10]. For example the central column of AUG is a strong source of carbon radiation, but as being fully coated by tungsten, not a net carbon source. Even surface sensitive investigations after a campaign yields only 10 mono-layers of carbon [11]. If a location, which acts as a significant source of carbon, is replaced by tungsten, the carbon deposition will be reduced. This 
was used to identify the low field side main chamber limiters and ICRH antenna limiters as strong carbon source [3].

Here we concentrate on the results of the 2002/03 campaign, which was carbon dominated, and the 2007 campaign with full tungsten PFCs. Parts of the main chamber had been tungsten coated before the 2003 experiments. In Fig. 1 a cross section of AUG including the tiles, which were analyzed is shown. Details of the analysis are reported in [3] and the results are summarized in Fig. 2 . The carbon- and hydrogen-deposition during the 2002/03 campaign are plotted at A, for the full tungsten PFCs in 2007 at B. The abscissa is given by the s-coordinate, which runs along the divertor tile surface. It starts at the inner divertor baffle via the private flux region to the outer divertor baffle. The numbering of the tiles is indicated at Fig. 1. During the 2002/03 campaign dominant deposition is found as a: $\mathrm{CH}$ layers at the inner divertor. The magnetically determined inner strike point was always located at tile 4 . Here up to $6 * 10^{19} \mathrm{at} / \mathrm{cm}^{2}$ carbon are found. Almost the same carbon deposition is on tile 5 , decreasing to $5 * 10^{18} \mathrm{at} / \mathrm{cm}^{2}$ at the baffle region (tile 6A). At the strike point module 4 the deposits form typical a: $\mathrm{CH}$ layers with $D / C \approx 0.4$. At the other inner divertor tiles a low hydrogen content $(D / C \approx 0.1)$ is observed. The jump of the hydrogen content from tile 4 to tile 5 correspond with the different materials used at the tiles. For historical reasons tile 4 was made out of CFC. Laboratory [12] investigations show that the hydrogen content of these layers depends strongly on the temperature of the substrate. So the higher hydrogen content of tile 4 can be attributed to the lower temperature or, more plausible, on the porosity of the CFC. At the private flux region (tiles 9 and 10) deposition is found close to the strike point region, indicating re-erosion and re-deposition as discussed below for the remote areas. The marker stripes at the outer divertor strike point module (tiles 1$)$ were destroyed, so no carbon deposition and erosion could be determined. On tiles 2 and 3 carbon erosion was 
found. In general the hydrogen content of the outer divertor is low, confirming that this region is erosion dominated. The only deposition is in the shadow region of tile 2, where the upper edge of this tile is shadowed by tile 3 for edge protection. Summing up all tiles a hydrogen inventory of $2.7 g$ is found for the $2002 / 03$ campaign.

The same measurement is shown for the 2007 campaign in Fig.2 B, which is expanded by more than a factor of 10. Carbon deposition is found at the inner baffle region, the private flux region and to a minor fraction at the outer divertor strike point module. The deposition at the outer divertor reflects the surface roughness of the VPS coatings used. Spectroscopy yields the carbon content of the SOL. Comparing the carbon and tungsten device the carbon deposition at the inner divertor drops by a factor of 15 . One would expect the carbon content in the SOL to behave similar, but only a reduction by a factor of 2 was found [14]. Hydrogen is deposited at the inner divertor and private flux region as a: $\mathrm{CH}$ layers $(0.4 \ll D / C \ll 1.0)$, similar as after the $2002 / 03$ campaign. Thermal desorption spectroscopy (TDS) measurements confirm this results at the inner divertor. At the outer divertor the situation is different. Whereas the signals obtained by nuclear reaction analysis (NRA) originate from the surface to a depth of about $6 \mu m$, the whole sample is measured by TDS. The higher results for TDS analysis compared to the NRA points to deeper penetration of deuterium into the VPS layers. For VPS layers this behavior is expected due to the porosity of the layers. Bulk tungsten was used in AUG only as Langmuir probes in the divertor. The of tungsten grade used for these probes was not specially selected for material investigations. Nevertheless NRA and TDS are applied to get the deuterium inventory. Whereas NRA investigations found only a small quantity, the total amount of deuterium determined by TDS is comparable to the one measured on the VPS layers. This hints to less deposition at the surface (due to the smoother surface), but also to implan- 
tation and deep diffusion of deuterium into the bulk tungsten. Nevertheless the total inventory found in bulk tungsten is almost the same as for the $200 \mu \mathrm{m}$ VPS layers [13]. In total $0.13 \simeq 0.20 \mathrm{~g}$ deuterium are found at the divertor tiles. To reduce the deuterium retained in AUG further the carbon content of the discharges has to be minimized. Possible carbon sources are electrical arcs, remnants from former campaigns, erosion by oxygen atoms and damages of electrical isolations. Consequently the PFCs had been additionally cleaned for the 2008 campaign.

\section{Deposition at remote areas}

As remote areas we subsume the areas below and behind the divertor tiles as well as the mounting structures. In AUG layers are found primarily below the roof baffle, indicated as $R e$ in Fig. 1. For safety purposes the hydrogen inventories of deposits at remote areas are especially critical, as they form hydrogen rich a: $\mathrm{CH}$ layers $(0.4 \ll D / C \ll 1)$ and a cleaning is hindered by the difficult access and the complicated structure. Small silicon wafers mounted at these different positions are used as probes for post mortem ion beam analysis. Additionally cavity probes, which allow to determine the effective sticking probability of the layergrowth preceding precursors [12] and quartz micro balance monitors, which give shot resolved information on the layer growth, are used [15]. From cavity probes and the decay length of the layer thickness it is deduced that the deposits are formed by activated precursors, which have a high sticking probability. Geometrical reconstruction from cavity probes yields the source of the precursors close to the strike point position.

The total amount of hydrogen deposited at the divertor region normalized to the total amount of hydrogen puffed during discharges is summarized in Table 1. During the campaign $2002 / 033.4 \%$ of the deuterium puffed during plasma 
discharges was found at the divertor. About $74 \%$ of this deuterium is deposited at the inner divertor, $21 \%$ at the remote areas. For the full tungsten device in 2007 only $0.7 \%$ of the deuterium input was found. Now $57 \%$ of the retained deuterium is found at the outer divertor. Only $28 \%$ is found in deposited layers at the inner divertor and $14 \%$ at remote areas. Half of the inventory at the outer divertor is located near the surface within the first microns. Whereas the deposition on the target plates changed strongly, the fraction of deposition at remote areas is almost constant.

The deposition at the target plates, derived from Fig. 2 and remote areas is plotted in Fig. 3. At remote areas small Si probes amounted at different locations are used to determine the deposition. In Fig. 3 the target plates are used as origin of the x-axis, the different probes are arranged by the distance to the target plates. At the inner divertor ( Fig. 3B ) the strongest deposition during a plasma discharge is found at the target plates. These layers are partly eroded again by the plasma in subsequent shots and form precursors, which build up layers at remote areas. As the origin of the precursors are the strike point areas an exponential decay towards the remote areas is expected, as observed. For the 2007 campaign the deposition at the target plates is reduced by a factor of 13 and the deposition in the remote areas is reduced by the same amount, the shape of the decay curve is not changed. At the outer divertor the behavior is more complicated. The re-erosion by the parasitic plasma below the roof baffle [15] was taken into account by the selection of the probe positions. For the 2003 campaign the deposits at the remote areas are an order of magnitude thicker than at the outer target plate. At a first glance this seems to be a contradiction to the origin of the precursors from the strike point tiles. A simple explanation is that most of the carbon deposited at the outer divertor target plates is re-eroded again, whereas at remote areas the erosion of the layers is negligible. This hints to the fact that most of the carbon deposited 
at the outer divertor is re-eroded again leading to a carbon gross-deposition much bigger than the net deposition derived from the layers at the end of the campaign. During the 2007 campaign the deposition at the remote areas is comparable to that of the target plates. The amount of deuterium at the target plate is similar for both campaigns. From post mortem analysis it is observed that deposition at the outer target plates are concentrated at specific locations. The layers are inside of valleys were they are hidden to the plasma ions, which hit the surface under a shallow angle [12]. The filling of the surface roughness may yield the same amount of deposition for both campaigns. Additional deposited layers at the outer target plate are eroded forming the deposits at remote areas. For the 2007 data the difference between gross- and net-depositions seems to be much smaller.

\section{Calibration of pumping and gas puffing systems}

The key point for an accurate gas balance is the calibration of the gas inlet and pumping systems. Pressure at AUG is measured at two different positions (see Fig. 1): at the midplane and in the divertor. The divertor gauge is located in between the cryo- and turbo pumps. As reference a highly accurate capacitive gauge with a full range of $13 \mathrm{~Pa}$ was used. The calibration before the experiments yields an absolute error of $0.04 \%$ at a pressure of $1 \mathrm{~Pa}$. After the campaign the same instrument was calibrated again, yielding an error of $0.07 \%$, i.e. the calibration did not change significantly during the experiments. To extent the pressure range of the gauge a stabilized ionization gauge was additional used. To take the variation of the gas composition into account, the ionization gauge is calibrated at the end of each discharge with respect to the capacitive gauge. All instruments are magnetically shielded for accurate measurement during a plasma

discharge. For the balance, particle input by various gas inlet valves $\Phi_{\text {valves }}$ and 
the NBI boxes $\Phi_{\text {Beam }}$ have been taken into account. As pumping systems the cryopump $\Phi_{C P}$, Turbo pumps $\Phi_{T M P}$ and NBI boxes $\Phi_{N B I}$ are considered.

To minimize systematic errors all relevant systems are calibrated with respect to the same instrument [16]. As first step the volume of the vessel was determined by expanding the content of a small volume into the closed vessel. A series of measurements leads to $V_{\text {vessel }}=41562 \pm 100 l$. For gas inlet piezo valves with internal feed back control are used [17]. The gas flow is monitored by the pressure sensor at the capillary tube. This value is used by the controller to regulate the opening of the piezo and at the same time it is recorded to monitor the injected amount of gas. The absolute amount of gas was calibrated by injection of a gas pulse into the known vessel volume. The pressure rise is measured by the midplane gauges. For calibration 12 different flow rates are applied and the results are fitted to a polygon. A former calibration done in 2003 has been repeated in 2008, leading to a correction of typical $2 \%$.

All pumping systems installed at AUG are limited by conductance, not pumping speed. The pressure during relevant plasma discharges at the divertor is in the range of some $0.1 \mathrm{~Pa}$, i.e. at the transition from molecular to fluid flow: the effective pumping speeds rises with pressure. The pumping speed was determined from the equilibrium pressure for a known gas inlet by the calibrated gas inlet system. Again several measurements for different pressures were done and the results are fitted to a straight line. This procedure was applied to the turbo pumps, the NBI boxes, the diagnostics and the in vessel cryopump. All results are summarized in Table 2.

The NBI pumping speed is determined with respect to the midplane pressure measurement, the turbo and cryopumps to the divertor gauges. As the cryopump is located in front of the pressure measurement, the correct conductance of the divertor has to be considered. The divertor structure in front of the cryopump is not 
completely tight, but has a conductance comparable to the divertor slits. During plasma operation, the pressure in the divertor dominates the midplane pressure. Only the conductance of the divertor slits is relevant for pumping during plasma operation. To derive the correct pumping speed, plasma discharges have to be used. Discharges with different divertor pressures are performed with closed gate valves to the turbo pumps. After the discharge, all gate valves are closed and the LHe penal of the cryopump was warmed up. The pressure rise determines the total amount of gas pumped by the cryopump. Unfortunately it was not possible to keep the divertor pressure constant during the whole discharge. For this reason the discharges were divided into time slices of typical $300 \mathrm{~ms}$. The average value of the pressure during these time slices was used to calculate the amount of pumped gas. Summing up all time slices the total amount of pumped gas was gained. Assuming a linear dependence of the pumping speed on the pressure, the pressure dependence was achieved from a fit of the total amount of gas pumped during the discharge.

Test runs with high gas flux and without plasma yield a total accuracy better than $1 \%$. This low value could not be reproduced after plasma discharges. Significant differences are found for a cold and warm $L N_{2}$ shielding of the cryopump. The gas temperature was identified as the dominant error. During experimental days the $\mathrm{LN}_{2}$ shielding of the in-vessel cryopump is always kept cold. On the other hand temperatures up to $400 K$ are measured by thermocouple at the limiters and some other areas 10 min after a high power discharge. Even higher temperatures are expected for some shielding of diagnostics, which are intentionally build with poor thermal contact to prevent a heating of the diagnostic parts. The neutral gas in AUG is not in thermal equilibrium after a plasma discharge. Recently Lipschultz showed that high accurate gas balance could be obtained in ALCATOR C-mod, if one operates only with the cryopump and measures the amount of 
pumped gas by the pressure rise after warming up of the pump [18]. This method implies a constant gas temperature, which is not given in AUG. The effective gas temperature was determined by puffing a known amount of gas and by measuring the additional pressure rise. This method leads to an error of about $3 \%$, in good agreement with the statistical errors reported below.

\section{Gas Balance measurements}

The standard conditioning of present fusion devices includes a wall coating as boronisation to introduce an additional getter in the main chamber [19]. Boron layers are used to getter oxygen but are also acting as a temporal storage for hydrogen. Before the 2008 campaign the PFCs were completely cleaned from former layers. No wall coating was used for plasma start up. Initial conditioning was done by baking and helium glow discharge (GD) cleaning only. From a vacuum point of view the most important material in AUG is still fine grain graphite: about $800 \mathrm{~kg}$ of carbon is used for the tiles. To remove water effectively from graphite a heating up to $350^{\circ} \mathrm{C}$ would be necessary. However, at AUG the maximum temperature allowed for baking of the vessel is $150^{\circ} \mathrm{C}$. Some water remains in the graphite tiles, which is released if the tile is heated by plasma discharges. The standard technique to reduce the plasma contamination by outgassing, wall coatings, was intentionally not used this time. As an alternative, plasma discharges were used to heat up PFCs and reduce the water content of the tiles. The outgassing was measured by residual gas analysis and even gas balances could be used to monitor the status of the PFCs. After about 100 plasma discharges no significant outgassing was observed anymore. Therefore we restrict our data base for the gas balance with tungsten wall to the phase after this conditioning and before the first boronisation of the 2008 campaign. The low amount of boron was 
confirmed by spectroscopy, which found that boron was below the detection limit during this phase [20].

Semi-detached H-mode discharges are foreseen to be the most relevant for ITER: we concentrate on high density discharges for the gas balance investigations. In AUG a standard H-mode discharge, which is performed on each experimental day, can be used as a basis for gas balances. This 1 MA shot is heated with $5 \mathrm{MW}$ of NBI, reaches line averaged densities of $9 \cdot 10^{19} \mathrm{~m}^{-3}\left(\approx 0.7 n_{g w}\right)$ and a total gas puff of $1 \cdot 10^{23}$ at during $6 \mathrm{~s}$. The temporal evolution of the gas balance for a typical shot of the series of standard shots is shown in Fig. 4. The gas input is the sum of the puffed gas $\Phi_{\text {valves }}$ and NBI flux $\Phi_{\text {Beam }}$. For the gas removed the flux to the pumping systems is summed up. The plasma inventory $N_{p l}$ is derived from the line averaged density and the plasma volume. Note that the plasma inventory is more than one order of magnitude less then the pumped gas.

For comparison the ratio $R_{D}$ was defined. This is build by the integral of the different pump fluxes during the time considered and the inventory of the plasma $N_{p l}$ and the neutral pressure in the vessel $N_{n t}$, normalized to the integral of the injected gas. The gas detained in the vessel is found as $1-R_{D}$.

$$
R_{D}=\left(\int\left(\Phi_{C P}+\Phi_{T M P}+\Phi_{N B I}\right) d t+N_{p l}+N_{n t}\right) / \int\left(\Phi_{\text {valves }}+\Phi_{\text {Beam }}\right) d t
$$

The discharge can be divided into different phases. The gas fluxes during these phases are summed up to calculate $R_{D}$ as shown in Table 3 . The plasma starts as a limiter discharge (lim). Only a minor gas puff of $6 * 10^{20} \mathrm{at} / \mathrm{s}$ is applied, outgassing of the wall is dominant, leading to $R_{D}>1$. For plasma density ramp up (ramp) the puffing is increased up to $3.3 * 10^{22}$ at $/ \mathrm{s}$. During this phase hydrogen is mostly absorbed by the wall, typical values are $R_{D} \approx 0.2$. After the high density phase is reached (high), the plasma density is constant. But $R_{D}$ is continuously rising as the pumping of the wall decreases until the PFCs are saturated. During 
this phase the wall is pumping till it is saturated. So the ratio $R_{D}$ is changing. Typical time scales are some seconds for strongly puffed discharges, whereas for low density discharges this phase lasts for the whole shot. As the wall pumping is reduced the neutral pressure at the divertor is rising, leading to a higher amount of pumped gas. For the discharge mentioned above steady state conditions are reached at $2.9 \mathrm{~s}(\mathrm{p}=\mathrm{p})$. During this phase all gas puffed is pumped within the error-bars of the measurements $\left(R_{D}=1\right)$. Obviously the wall is saturated and is not acting as pump anymore. For the ramp down (ramp-dwn) the gas input is reduced at $4.1 \mathrm{~s}$. Strong outgassing starts immediately, leading to $R_{D} \approx 10$. The long term outgassing is discussed below.

Additionally, the data for the whole plasma discharge, i.e. the time slice with plasma current $I_{p} \geq 200 \mathrm{kA}$ and the $15 \mathrm{~s}$ after the start of the discharge (short term) are added. To compare discharges with different densities, which may not reach steady state conditions, the shot integrated values are needed. The criterion on the plasma current allows automatically evaluation of shots, even if they ended with a disruption. As disruptions in AUG are routinely mitigated by massive gas puff, gas balance including this phase will yield misleading results. The time slot till $15 \mathrm{~s}$ is useful to compare with the former experimental campaign on AUG, where the data collection system was restricted to this period.

To get more representative results the average values on all standard $\mathrm{H}$ - mode shots of the 2008 campaign after the initial conditioning and before the first boronisation are considered. The standard $\mathrm{H}$-mode discharge is performed on each experimental day, which result in a data base of 30 discharges. Although the discharge program used is basically always the same, small variations on the standard shots result from the use of this shot for piggy back programs. Averaging theses discharges leads to a retention of $1-R_{D}^{\text {shot }}=8.2 \pm 3.3 \%$ for the whole discharge and $1-R_{D}^{p=p}=1.5 \pm 3.5 \%$ for the steady state phase. The statistical error of 
$3 \%$ obtained from this database agrees with the expected uncertainty due to the gas temperature.

To reach the steady state phase it is necessary to saturate the PFCs. Wall saturation requires high gas puffing rates, and is in AUG only reached for high density shots. Taking the same data base the amount of gas needed to fill up the wall could be derived ( Fig. 5). Typical $4.1 \simeq 6.5 * 10^{22}$ at of hydrogen are needed to be puffed to reach the steady state phase $(\mathrm{p}=\mathrm{p})$. The significant variation of this value is caused by different plasma conditions as for example different ELM behavior. Calculating the gas retained in the wall $19 \pm 1 * 10^{21}$ at hydrogen is found. Note that this value shows much less variation than the amount of gas puffed. This hints to the fact that the wall loading is almost independent from details of the discharge. If we assume that a significant amount of gas is pumped by the PFCs at the main chamber (in reality the surface may be larger as the tile sides and the vessel ports may also play a role) the 'layer density' of the adsorbed gas can be calculated and the gas retained is equal to 44 monolayer hydrogen. For pure adhesion the layers are to thick, indicating solution of $\mathrm{H}$ in the near surface tungsten. This idea is supported by the typical outgassing time of some minutes [6].

The retention ratio $R_{D}$ taken for the whole plasma discharge (shot) for all plasma discharges during the 2008 campaign after initial conditioning and before the first boronisation is plotted in Fig. 6. The first finding is that no discharges with $R_{D}>1$ are found, i.e. even for high heating powers no significant discharge integrated outgassing from the wall is observed. This hints to the fact that before the start of the discharge no significant inventory is bound to the surface. Details on this issue will be discussed in the section on long term outgassing. The general behavior is a strong decrease of $R_{D}$ for low gas fluences. For higher fluences the $R_{D}$ approaches asymptotically the $R_{D}=1$ line. As $R_{D}$ is calculated only during 
the shot the outgassing after the discharge is not taken into account here, leading to a retention for all shots. As for wall saturation a constant value is needed, the $R_{D}$ value gets closer to one for high amounts of gas puffed. After boronisation, shots with gas release are observed again, as usual in many other devices [18] suggesting the storage of hydrogen in the boron layers.

\section{Gas Balance with Carbon Wall}

Gas balances with carbon wall were reported in [21]. In the view of the new and more accurate calibration the data have been re-evaluated. The absolute values differ by less than $10 \%$, confirming the former results. Unfortunately the new calibration is not completely valid for the older data, as parts of the gas inlet system and the outer divertor had been changed in between. During the carbon phase AUG was always operated with boronised PFCs. A plot similar to Fig. 6 shows, especially for low density, strongly heated scenarios significant outgassing from the wall [21]. We suppose that this behavior is mostly due to the boron layers, as a similar behavior is observed for boronised tungsten wall. This may be also true for all carbon based devices since to our best knowledge no high quality data for pure carbon PFCs exist in the literature. Even if no boronisation was applied, boron layers of some micron thickness remain from former campaigns [22], [23].

For direct comparison the same discharges with tungsten PFCs as for carbon PFCs would be ideal. Due to a damage of one power generator of AUG it had not been possible to repeat former discharges without modification. The total puffing rate was identified as most important parameter. For comparison a discharge as similar as possible to the present standard H-mode discharge was selected. Applying the data evaluation and calibration to the older data bigger error bars has 
to be accepted. To compare the time depended behavior for different PFCs, the retained amount of gas $\Phi_{\text {ret }}$ was calculated from the difference of the gas input $\left(\Phi_{\text {valves }}+\Phi_{\text {Beam }}\right)$ and removed gas $\left(\Phi_{C P}+\Phi_{T M P}+\Phi_{N B I}\right)$. These values are plotted for a similar discharge with carbon and tungsten PFCs in Fig. 7. Additionally the balance defined by the integral of $\Phi_{\text {ret }}$ is shown. The abscissa is shifted slightly, so that the ramp down phases of both discharges overlay. For both materials the discharges starts with a phase which loads the wall. For the tungsten shot the puffing was optimized for fast plasma density build up, which saturates the wall quite fast. After wall saturation a phase with no additional wall loading is reached from 4.2 till $5.4 \mathrm{~s}$. A similar amount of gas was puffed for the carbon shot. The $\Phi_{r e t}$ for carbon wall drops after the initial phase, but did not reach zero during the discharge. A slight decrease indicate that the wall pumping is less at the end of the flat top. A similar behavior is found in gas balances for JET [24], but it is unclear, if it is possible to saturate a carbon wall completely. Typically $5 * 10^{21} \mathrm{at} / \mathrm{s}$ are retained during a high density discharge. During the plasma ramp down both discharges show similar behavior.

\section{Long Term Retention}

In general, the measurement of long term outgassing is hindered by the operation scheme of AUG. As the inventory of the in-vessel cryopump is restricted by safety reasons, regeneration is needed after each high density shot. Due to this the outgassing could be only determined until typically $100 \mathrm{~s}$ after the discharge. One example with a delayed regeneration after a high density discharge is shown in Fig. 8. The data gap at $45 \mathrm{~s}$ is due to the closing of the NBI valves, which changes the pumping speed. A base vacuum of $2.7 * 10^{-5} \mathrm{~Pa}$ equal to a pumped flux of $4 * 10^{18} \mathrm{at} / \mathrm{s}$, as measured before the shot, was subtracted. The pressure curve 
could be fitted by using two exponential decays: the first with a time constant of $22 \mathrm{~s}$, the second one with $138 \mathrm{~s}$ decay time. Integrating the curve, the total amount of gas release during the long term outgassing could be measured. The results for similar shots ( $1 \mathrm{MA}, \approx 7.3 \mathrm{MW}$ auxiliary heating, $n_{e} \approx 1 * 10^{20} \mathrm{~m}^{-3}$ ) with tungsten and carbon PFCs are compiled in the Table 4. The total amount of puffed hydrogen is quite similar for both shots. For this type of discharge $73 \%$ of the puffed gas is pumped during plasma build up and flat top for the tungsten PFCs. This value is only $64 \%$ for the carbon PFCs, reflecting that no phase with saturated wall occurs. Outgassing of the wall starts during the shot, as the gas puffing rate is reduced during plasma ramp down to avoid density limit disruptions. During the plasma ramp down another $17 \%$ of the gas is released for tungsten PFCs, i.e. only $10 \%$ of the puffed gas is retained in the vessel at the end of the discharge. For carbon PFCs the release during ramp down is less, resulting to an inventory of $23 \%$ of the puffed gas. The first seconds after the discharge show strong outgassing for both wall materials. For tungsten PFCs almost all the puffed gas is released within one minute. The long term outgassing phase is monitored until $650 \mathrm{~s}$. Another $3 \%$ of the puffed gas is measured during this time, leading to an overall balance of $103 \%$, somewhat higher than the puffed gas. The reason can be wall heating or, more realistically, the limited accuracy of the measurements. In summary this means that most of the puffed gas is pumped during the plasma operation for tungsten PFCs. The strongest outgassing occurs during the plasma ramp-down. The fast decay time constant of $20 \mathrm{~s}$ right after the discharge leads to a removal of most of the gas within some minutes. Probe data, as mentioned above found a deposition of $0.7 \%$ of the puffed gas for the 2007 campaign [3]. Showing that both methods agree within the error bars. For carbon PFCs a significant amount of hydrogen is missing after $15 \mathrm{~s}$. Unfortunately, the long term outgassing was not measured as it was blended by an automatically started HeGD. 
Residual gas analysis during the HeGD are hampered by the simultaneous regeneration of the in vessel cryopump. Former investigations yielded that about $10 \%$ of the puffed gas was released as hydrocarbons during HeGD and another $10 \%$ on a time scale of a day [21]. This value is an average on different shot scenarios and has consequently a bigger error bar. Probe measurements of deposits yielded a deposition of $3.4 \%$ of the hydrogen puffed during the whole campaign with predominantly carbon PFCs. Keeping the error-bars of the gas-balance measurements after the discharge in mind, again a good agreement is found.

The low hydrogen wall inventory for tungsten PFCs is confirmed by the plasma operation: In contrast to the carbon wall, almost no GD cleaning is needed in AUG for normal plasma operation anymore. For carbon PFCs strongly heated low density scenarios were performed without gas puff: the hydrogen was released from the walls. For tungsten PFCs no wall inventory is available anymore as seen in Fig. 6.

\section{Summary and discussion}

A combination of deposition measurements and gas balances is used to investigate the hydrogen retention in a full tungsten fusion device. Only $0.7 \%$ of the puffed during a campaign puffed hydrogen as measured by the probes. The majority of the hydrogen is bound in a: $\mathrm{CH}$ layers, as carbon is still an important impurity in AUG. The carbon content is determined by the fact, that in AUG coatings of tungsten on carbon tiles are used. Another important sink of hydrogen is deep implantation inside the tungsten layers at the outer divertor. The amount of hydrogen bound in tungsten depends strongly on the kind of tungsten used and needs further investigations. A direct comparison of probe and gas balance techniques is hampered, as the permanent deposition is too low for the accuracy reached by 
the gas balance technique.

Gas balances are used to establish the technique needed during the hydrogen and deuterium phase of ITER, to evaluate an operation scheme with low permanent wall retention and to extrapolate to ITER. Whereas the probe technique yields an average on all kinds of discharges the gas balance can be used to investigate different discharge scenarios. We assume that the typical operation of ITER will be at high gas fluxes and the PFCs will be made out of tungsten. During the long term discharges the wall saturation regime will be reached. Assuming the same behavior as in AUG and a 10 times bigger geometrical surface, an inventory of $0.5 \mathrm{~g}$ hydrogen is expected for wall saturation. This amount of gas will be mostly released during plasma ramp down and within 15 minutes after the discharge. After wall saturation a steady state phase will occur. However, the increase of the additional inventory cannot be extrapolated from the ASDEX Upgrade results because the wall temperatures in ITER will be much higher and the measurement errors of the gas balance in AUG - although already quite small - are still too large.

Probe and gasbalance measurements will be continued in AUG. For the 2009 campaign the boron layers will be removed again. Plasma operation will be started without wall coating and the damaged power generator will be back again. This will allow to extent the shot length and to operate in different kind scenarios. To enhance the accuracy of the gas balance the installation of a separate volume in front of the roughing pumps is planed. This will allow to measure the pumped gas at a known temperature and higher accuracy.

\section{References}

[1] Neu, R. et al.,"Final steps to an all tungsten divertor tokamak", J. Nucl. Mat., 363- 
365, (2007), 52 .

[2] Neu, R. et al.,"Plasma wall interaction and its implications in an all tungsten divertor tokamak", PPFC, 49, (2007), B59.

[3] Mayer, M. et al., "Carbon balance and deuterium inventory from carbon dominated to a full tungsten ASDEX Upgrade”, J. Nucl. Mat, 390-391, (2009), 538.

[4] Roth, J. et al.,"Tritium inventory in ITER plasma-facing materials and tritium removal procedures“,PPCF, 50, (2008), 103001.

[5] R.A.Causey, "Hydrogen isotope retention and recycling in fusion reactor plasmafacing components “, J. Nucl. Mat, 300,(2002), 91.

[6] Schmid, K. et al., "The implications of high-Z first-wall materials on noble gas wall recycling”, Nucl. Fusion, 47, (2007), 984.

[7] Mayer, M. et al.,"The Deuterium Inventory in ASDEX Upgrade”, Nucl. Fusion, 47, (2007), 1607.

[8] J.Likonen, et al.,"Beryllium accumulation at the inner divertor of JET", J. Nucl. Mat., 337-339, (2005,) 60.

[9] Mayer, M. et al.,"Erosion of tungsten and carbon markers in the outer divertor of ASDEX Upgrade",Physica Scripta, T128, (2007), 106.

[10] Puetterich, T. et al.,"Carbon influx studies in the main chamber of ASDEX Upgrade",PPCF, 45, (2003), 1873.

[11] Kallenbach, A. et al., "Spectroscopic investigation of carbon migration with tungsten walls in ASDEX Upgrade”, J. Nucl. Mat, 363-365, (2007), 60.

[12] Mayer, M. et al.,"Further insight into the mechanism of hydrocarbon layer formation below the divertor of ASDEX Upgrade",Nucl.Fusion, 46, (2006), 914. 
[13] Sugiyama, K. et al., "Deuterium inventory in the full tungsten divertor of ASDEX Upgrade", submitted to Nucl. Fusion

[14] Kallenbach, A. et al., "Non-Boronized operation of ASDEX Upgrade with fulltungsten plasma facing components”,Nucl. Fusion, 49, (2009) 045007

[15] Rohde, V. et al.,"Carbon erosion and a:C-H layer formation ”,J. Nucl. Mat., 337339, (2005), 104.

[16] Rohde, V. et al.,"Gas balance in ASDEX Upgrade with tungsten wall”, J. Nucl. Mat., 390-391, (2009), 474.

[17] Bates S.C. and K.H.Burrell, "Fast gas injection system for plasma physics experiments”, Rev. Sci. Instr. 55 (1984), 934.

[18] Lipschultz,B., et al.,"Hydrogenic retention with high-Z plasma facing surfaces in Alcator C-Mod“, ,Nucl.Fusion, 49, (2009), 045009.

[19] Rohde, V. et al.,"Wall conditioning in ASDEX Upgrade”,J. Nucl. Mat., 363-365, (2007), 1369.

[20] Dux, R. et al., "Plasma wall interaction and plasma behaviour in the non-boronised all tungsten ASDEX-Upgrade”, J. Nucl. Mat., 390-391, (2009), 858.

[21] Mertens, V. et al., "Hydrogen Gas balance in ASDEX Upgrade with divertor II b", ECA ,27A, (2003), P-1.128.

[22] West, W. P. et al., "Plasma Operation and Monitoring of Wall Conditions on DIIID Over Extended Periods Between Boronizations”, J. Nucl. Mat, 390-391, (2009), 461.

[23] Pegourie,B, et al., "Overview of the Deuterium inventory campaign in Tore Supra: operational conditions and particle balance", J. Nucl. Mat, 390-391, (2009), 550. 
[24] Loarer, T. et al.,"Gas balance and fuel retention in fusion devices“, Nucl. Fusion, 47, (2007), 1112. 


\begin{tabular}{|c|c|c|c|c|c|c|}
\hline year & PFCs & Total & inner & outer & remote & \\
\hline $2002 / 03$ & C & $3.4 \%$ & $2.5 \%$ & $0.2 \%$ & $0.7 \%$ & {$[7]$} \\
\hline 2007 & W & $0.7 \%$ & $0.2 \%$ & $0.4 \%$ & $0.1 \%$ & {$[3]$} \\
\hline
\end{tabular}

Table 1: Deuterium inventory normalized to the gas input found at the inner and outer divertor PFCs and remote areas. 


\begin{tabular}{|c|c|}
\hline Pump & $D_{2}$ pumping speed $[1 / \mathrm{s}]$ \\
\hline & $0.001 P a \leq p \leq 1 P a$ \\
\hline Turbomolecular Pumps & $16100-6131 \cdot p+1835 \cdot p^{2}$ \\
\hline Cryo pump & $115000+276600 \cdot p$ \\
\hline NBI Box 1 & $28350+46230 \cdot p$ \\
\hline NBI Box 2 & $24660+31450 \cdot p$ \\
\hline Diagnostics & $\leq 500$ \\
\hline
\end{tabular}

Table 2: Pumping speeds for deuterium of the systems used at AUG. The pressure dependence is due to the limited conductance to the pumps. 


\begin{tabular}{|c|c|c|c|c|c|c|}
\hline Phase & $T_{\text {start }}$ & $T_{\text {end }}$ & $N_{\text {in }}$ & $N_{\text {out }}$ & $\Delta N_{p l}$ & $R_{D}$ \\
\hline & $\mathrm{s}$ & $\mathrm{s}$ & $10^{20} a t$ & $10^{20} a t$ & $10^{20} a t$ & \\
\hline limiter & 0.0 & 0.3 & 2.2 & 0.7 & 2.2 & 1.35 \\
\hline ramp-up & 0.3 & 1.2 & 60 & 1.1 & 7.2 & 0.14 \\
\hline high dense & 1.2 & 4.1 & 799 & 690 & 2.5 & 0.87 \\
\hline $\mathrm{p}=\mathrm{p}$ & 2.9 & 4.0 & 339 & 340 & 0.6 & 1.01 \\
\hline ramp-dwn & 4.1 & 6.5 & 19 & 164 & -0.7 & 8.05 \\
\hline shot & 0.35 & 6.2 & 915 & 869 & - & 0.95 \\
\hline short term & -5 & 15.5 & 947 & 932 & - & 0.98 \\
\hline
\end{tabular}

Table 3: Gas balance for the different phases of the standard H-mode shot 22974. 


\begin{tabular}{|c|c|c|c|c|}
\hline & 22974 & & 17308 & \\
\hline Phase & $10^{20} a t$ & $\%$ & $10^{20} a t$ & $\%$ \\
\hline \hline Total Puff & 947 & & 907 & \\
\hline \hline Total Pumping & 973 & 103 & 782 & 81 \\
\hline Normal Plasma & 692 & 73 & 578 & 64 \\
\hline ramp down & 164 & 17 & 114 & 13 \\
\hline Pumping 15 s & 76 & 8 & 49 & 5 \\
\hline Pump long term & 41 & 4 & $?$ & $?$ \\
\hline Probes found & & 0.6 & & 2.7 \\
\hline
\end{tabular}

Table 4: Long Term Retention for tungsten (\#22974) and carbon (\#17398) PFCs.

The values are also shown normalized with respect to the total gas input. 


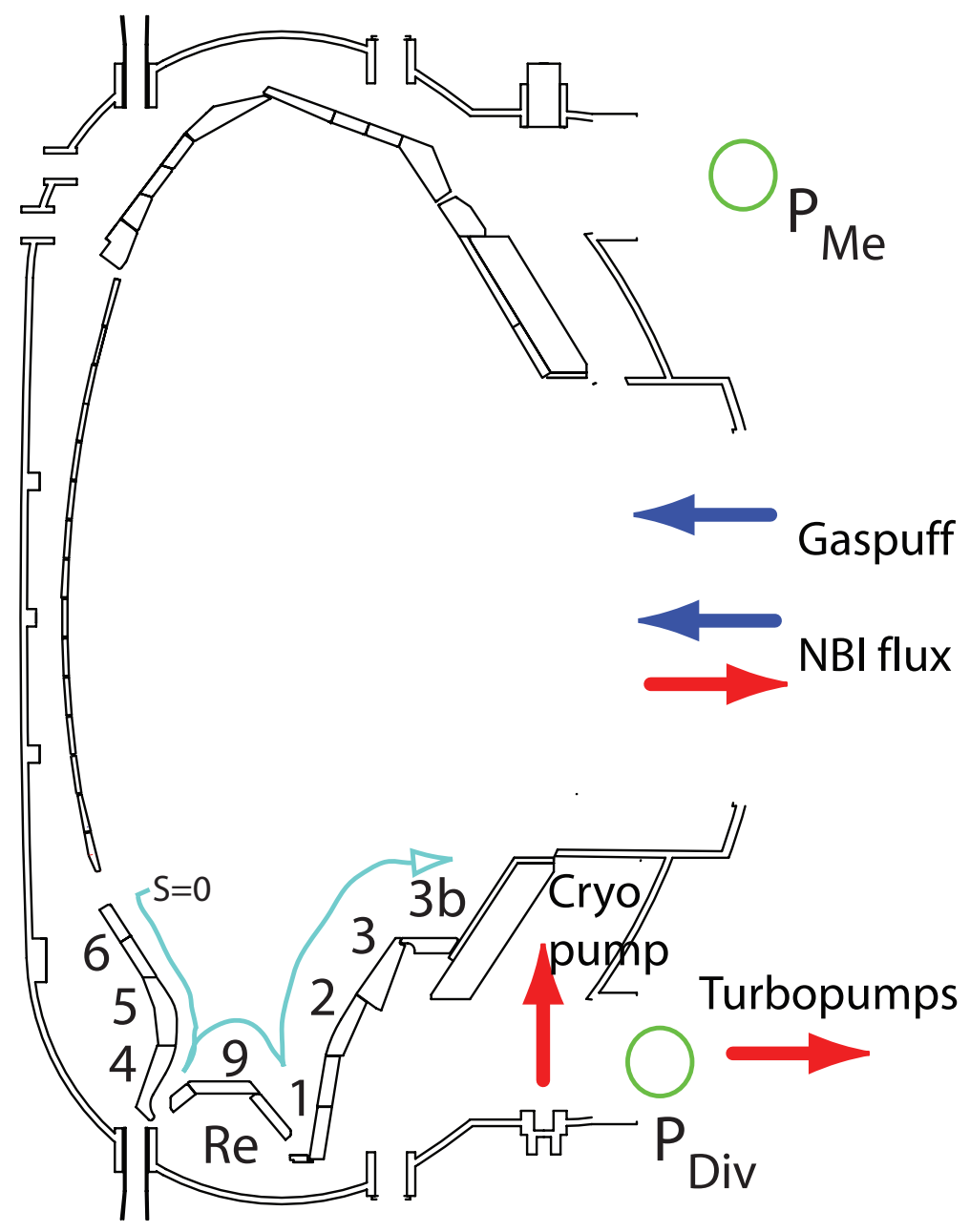

Figure 1: Position of the tiles (1-9) and remote areas (Re) used for post mortem analysis. The pumps and gauges are also indicated. 


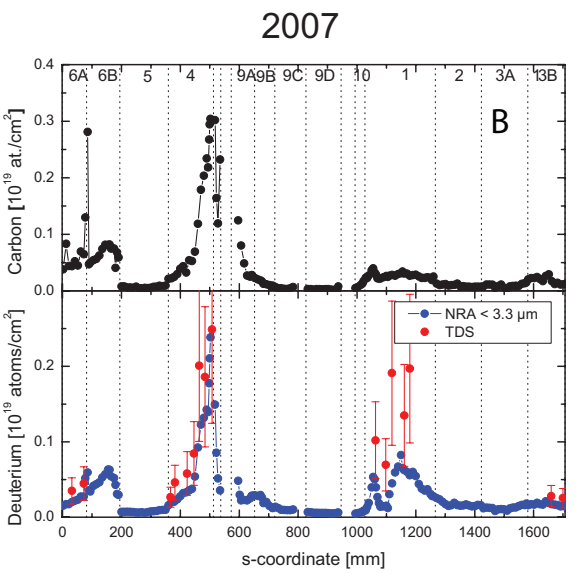

Figure 2: Carbon and deuterium inventory of the divertor tiles with carbon (A) and tungsten (B) PFCs. Note the different scales for carbon and tungsten campaigns. Negative numbers represent net erosion. For the 2007 campaign results of TDS measurements are added in red. 

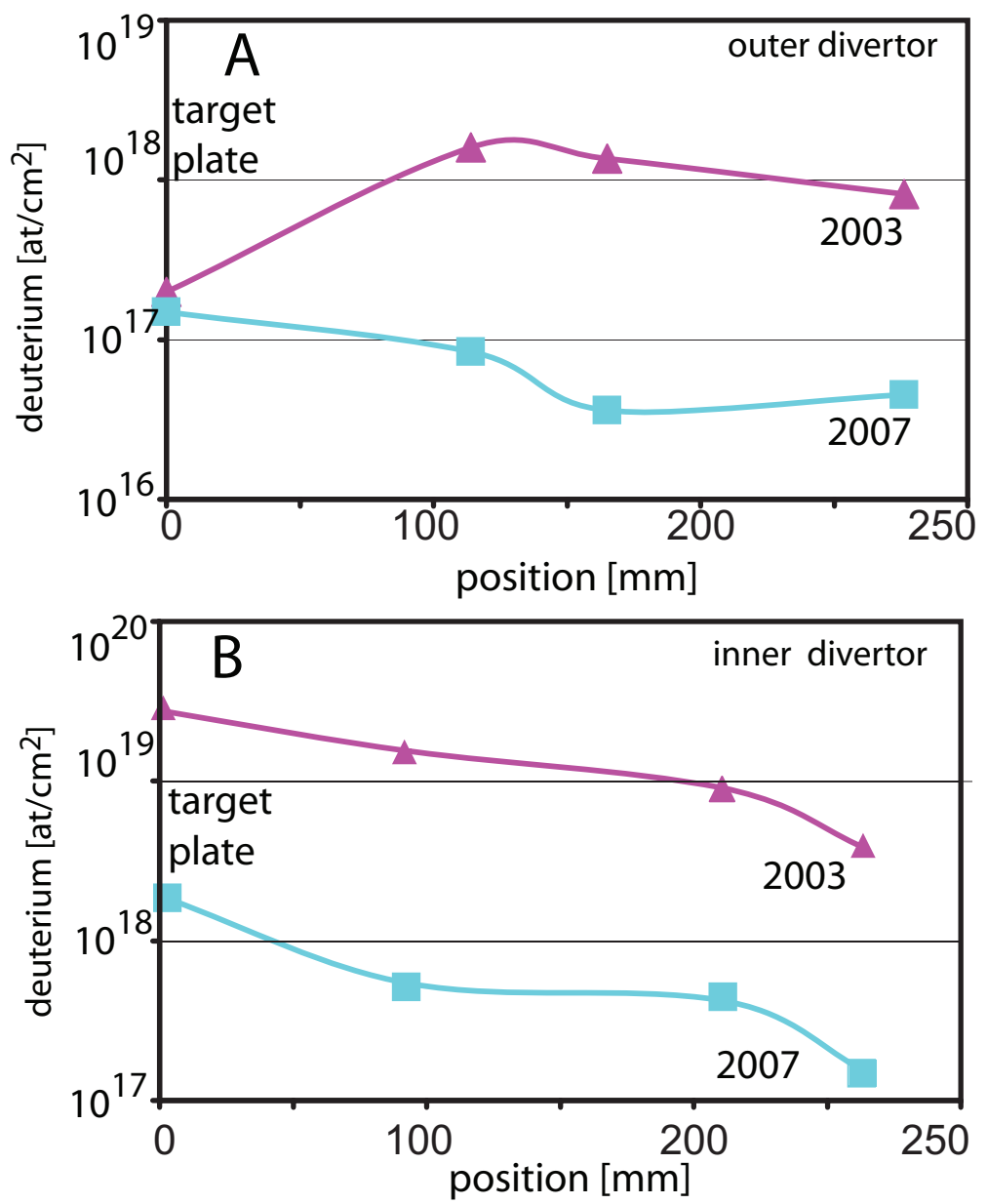

Figure 3: Deuterium deposition below the divertor structure as a function of the distance from the divertor plates. The results are given in magenta (2003) and cyan (2007). 


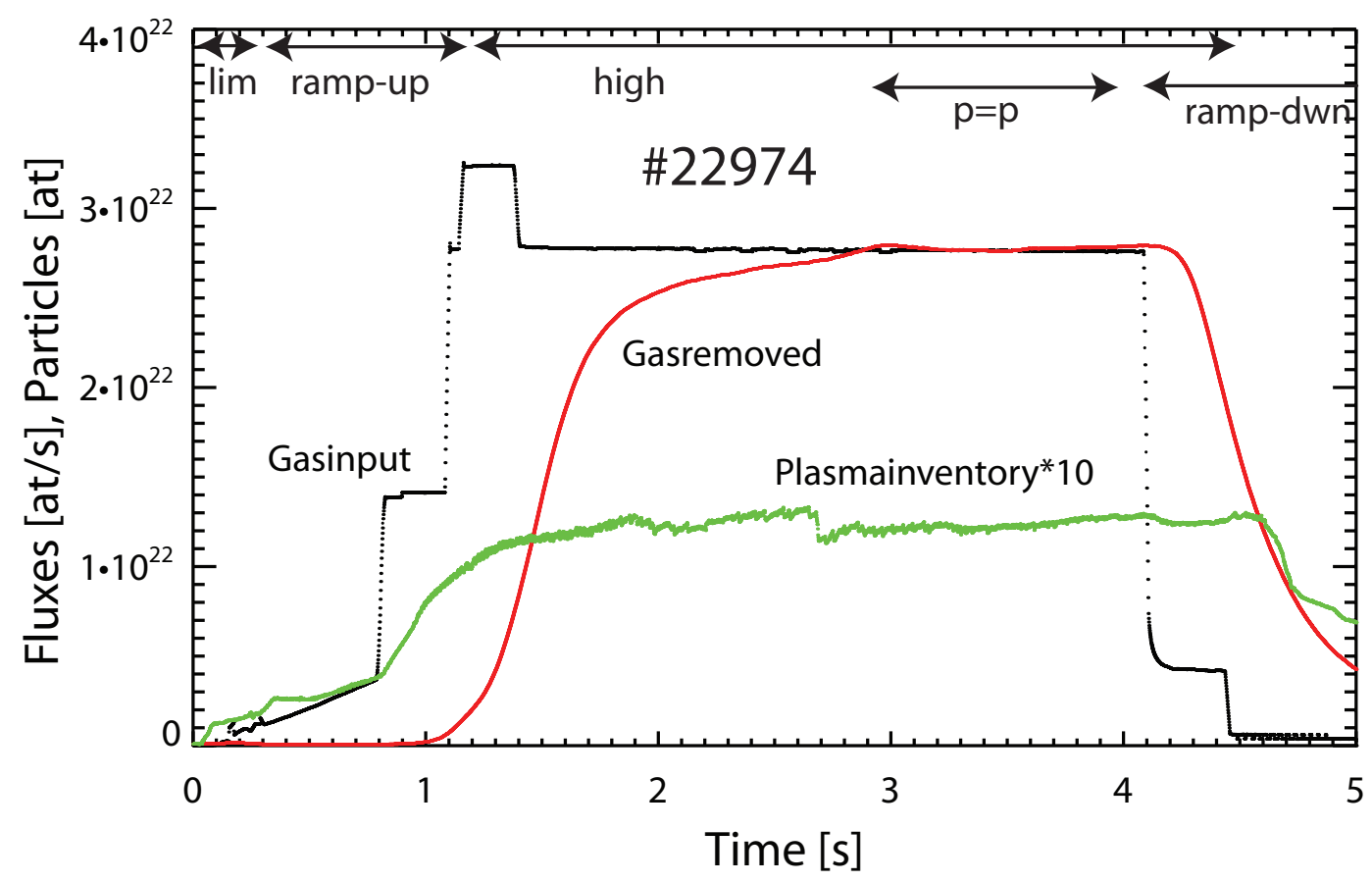

Figure 4: Gas input and removed gas for a standard H-mode shot. The different phases of the discharges, as used in table 2 are indicated. 


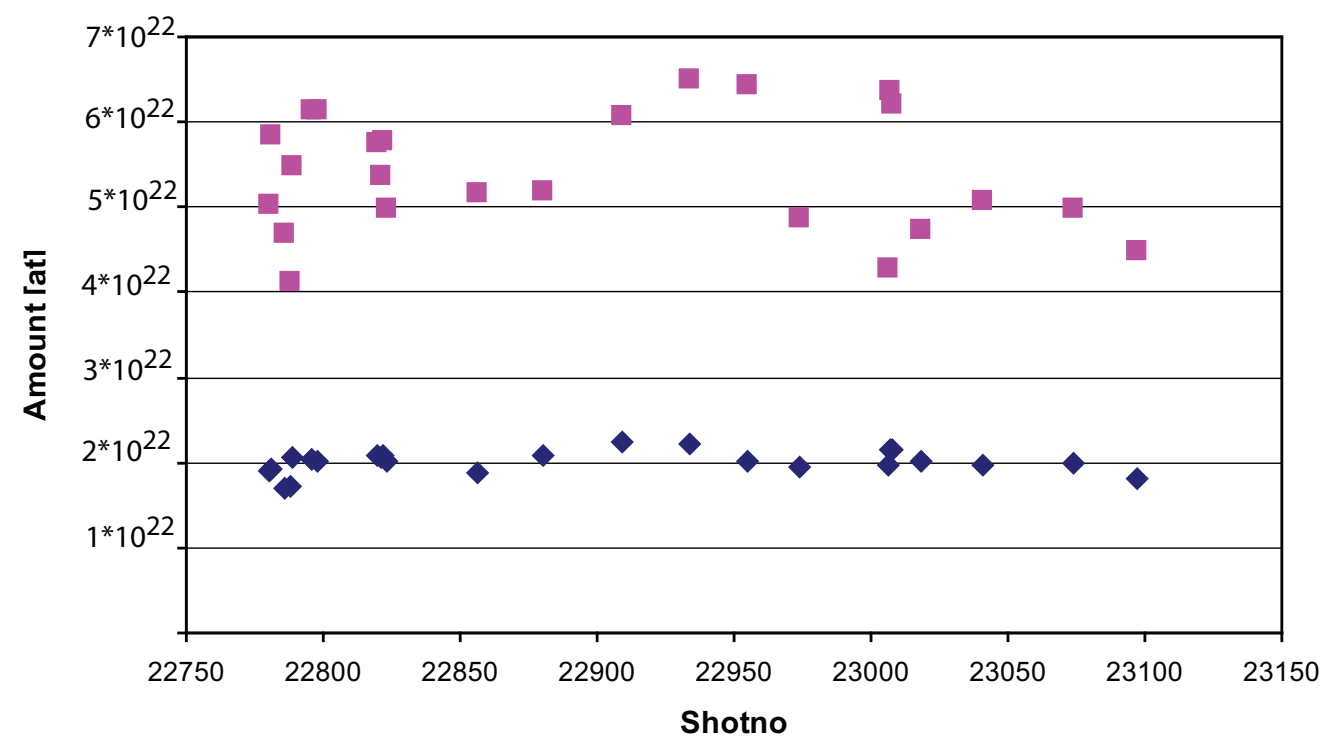

Figure 5: Gas input needed to reach the steady state phase (magenta squares). The amount of retained gas until reaching steady state is plotted in blue diamonds. 


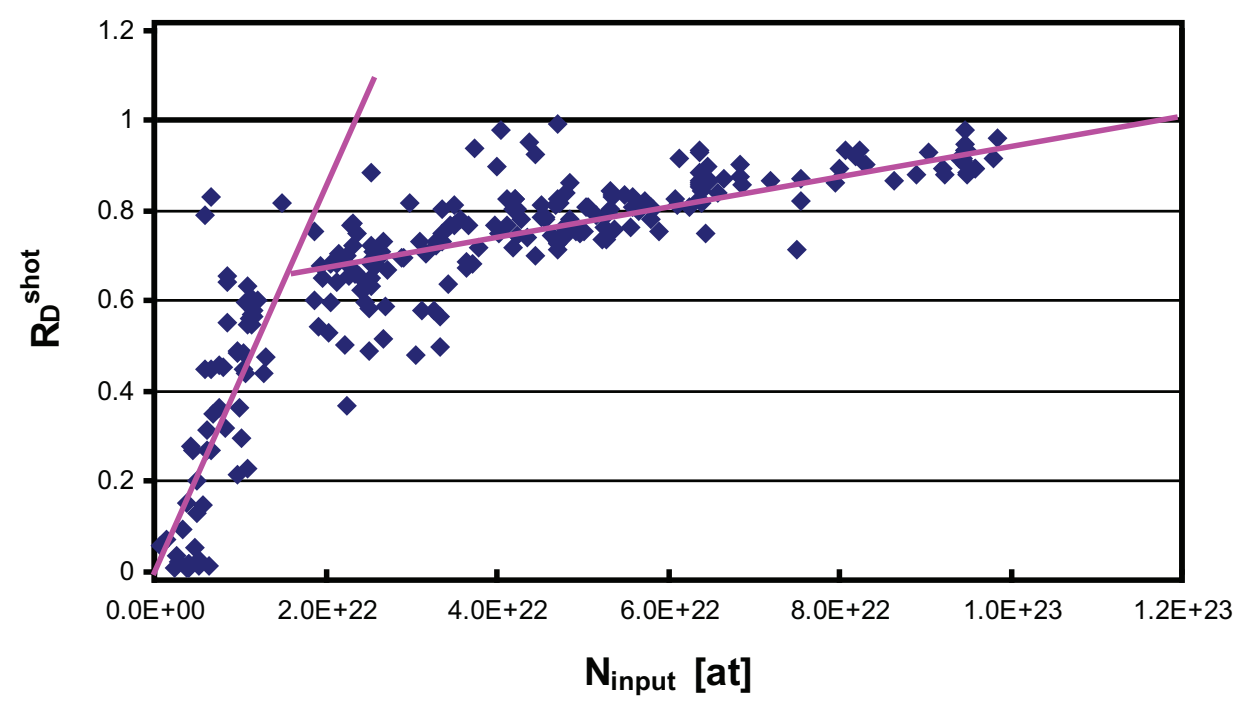

Figure 6: $R_{D}^{\text {shot }}$ for all discharges of the 2008 campaign after the initial conditioning and before the boronisation. 


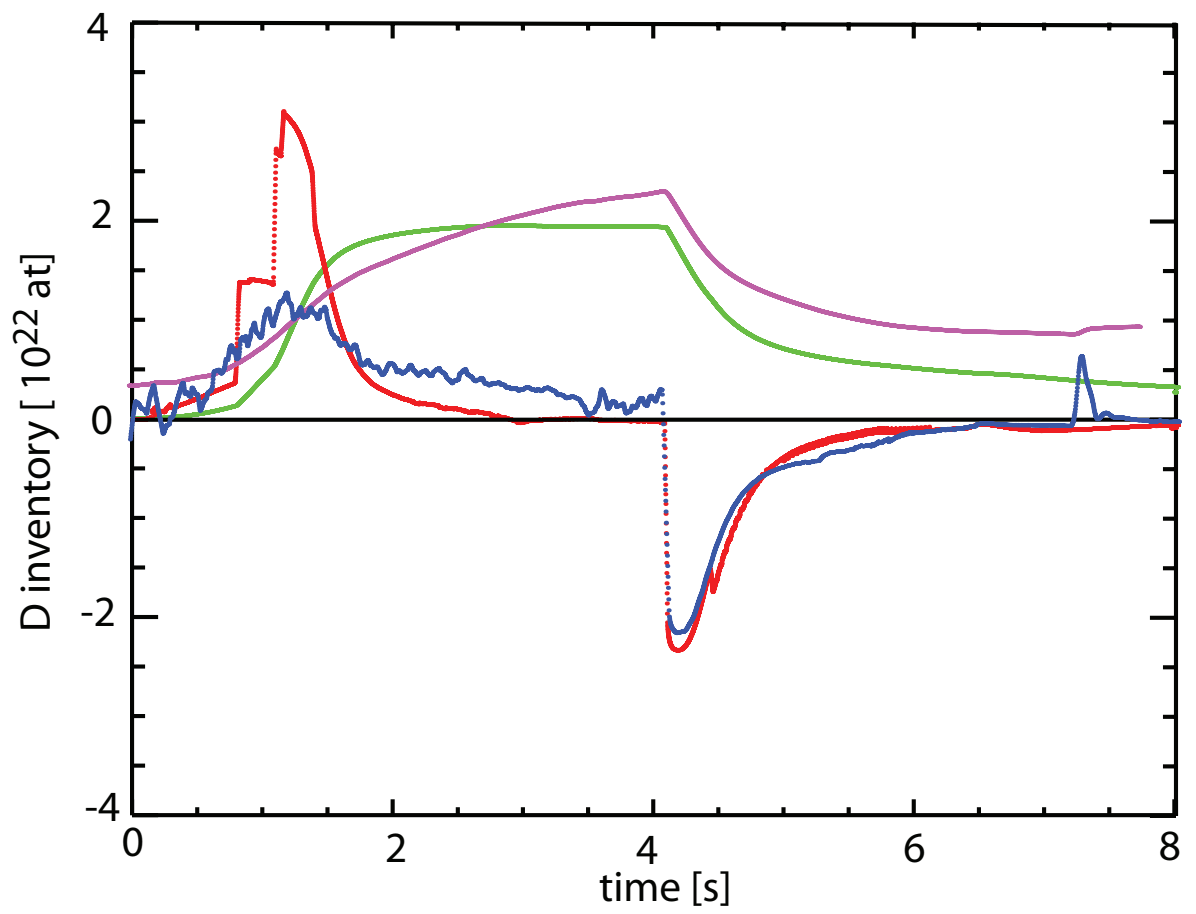

Figure 7: Comparison of the gas balances for carbon (\# 17308) and tungsten wall (\# 22974). The difference of gas puff and removal is shown in red (W) and blue (C). The integral in green (W) and magenta (C). 


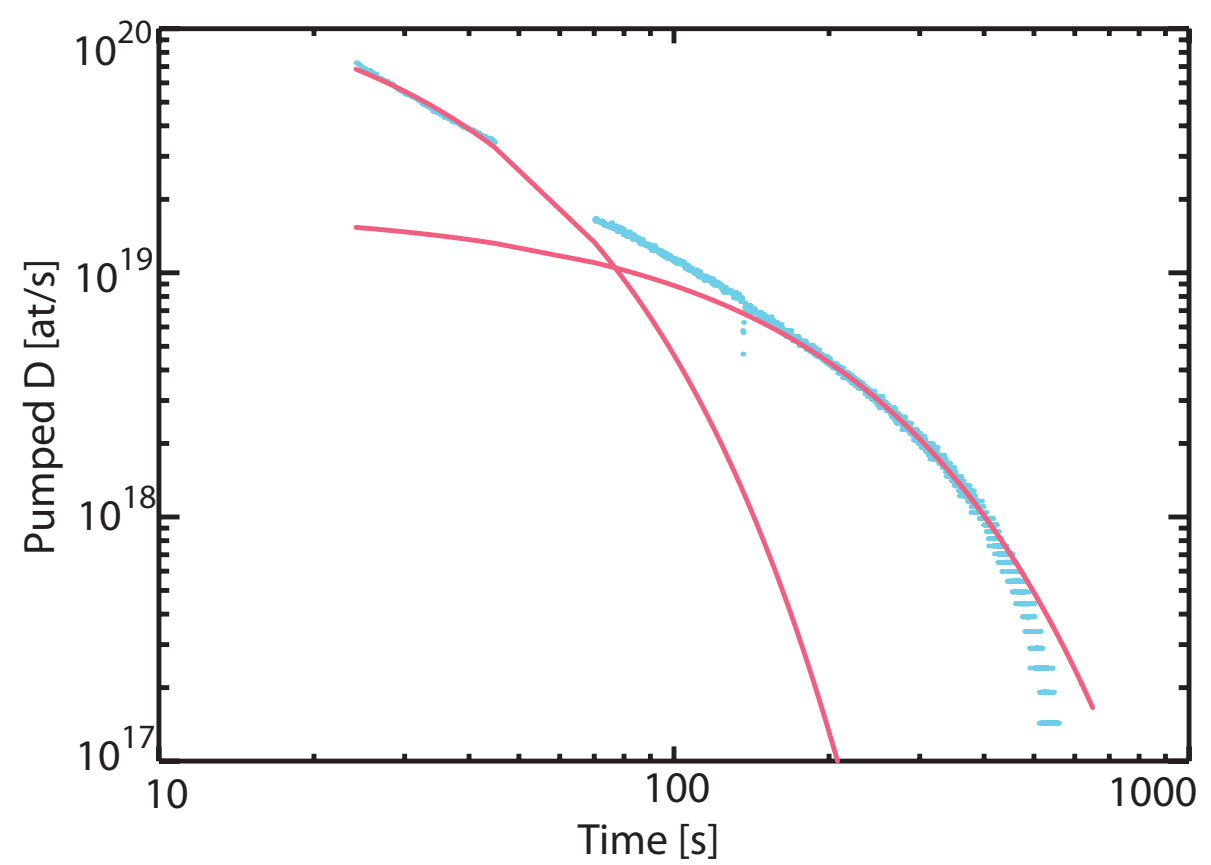

Figure 8: Gas removed after a discharge with tungsten PFCs. Exponential functions are fitted to the time slices $25-40 \mathrm{~s}$ and $200-400 \mathrm{~s}$. 